\title{
Fármacos e Doenças Crônicas em idosos hipertensos e/ou diabéticos praticantes de exercícios físicos
}

\author{
Medications and chronic diseases in diabetic and/or \\ hypertensive elderly practitioners of physical exercises
}

Fármacos y Enfermedades Crónicas en ancianos hipertensos y / o diabéticos practicantes de ejercicios

físicos

Giselly Cândido

Bruna Mastroldi dos Santos

Paulo Roberto Rocha Júnior

\begin{abstract}
RESUMO: O objetivo foi analisar a incidência de doenças crônicas não transmissíveis (DCNT) e a utilização de fármacos em idosos hipertensos e/ou diabéticos praticantes regulares de exercício físico. Como método, o estudo de campo, epidemiológico e transversal sobre DCNT e a utilização de fármacos em indivíduos com 60 anos ou mais de idade, praticantes regulares de exercício físico e diagnosticados com Hipertensão Arterial Sistêmica (HAS) e/ou Diabetes Mellitus (DM). Avaliaram-se 223 idosos, sendo 14,8\% ( $\mathrm{n}=33$ ) do sexo masculino e 85,2\% ( $n=190)$ do sexo feminino. A idade média foi de $69,8 \pm 6,9$ anos. Em relação à frequência semanal dos exercícios, observou-se que $57,8 \%$ os praticavam de 1 a 2 vezes por semana; 33,6\%, de 3 a 4 vezes; e 8,5\%, de 5 a 7 vezes na semana. Quanto ao uso de fármacos, 142 tipos foram relatados, dentre estes, destacaram-se os anti-hipertensivos, hipolipemiantes, antidepressivos, psicotrópicos cerebrais, hipoglicemiantes e supressores de absorção óssea. Dentre as DNCT investigadas, destacaram-se o DM, a HAS, as dislipidemias e o hipotireoidismo. Na correlação entre o tempo de exercício físico e o número de fármacos pôde-se observar forte correlação estatística $(\mathrm{r}=0,0848)$, enquanto para as DCNT, esta correlação foi moderada ( $\mathrm{r}=0,0593)$. Concluiu-se que, com o envelhecimento, observa-se um aumento da prevalência de DCNT, uma diminuição da prática de exercício físico, e um aumento no consumo de fármacos. Foi demonstrada uma maior adesão do sexo feminino na prática de exercício físico, mas quanto maior a idade, menor a adesão, e que os idosos praticantes de exercício físico por mais tempo consumiam menos fármacos e apresentavam menos DCNT.
\end{abstract}

Palavras-chave: Idoso; Exercício; Doença Crônica; Uso de medicamentos. 
ABSTRACT: The objective was to analyze the incidence of chronic noncommunicable diseases (NCDs) and the use of drugs in hypertensive elderly and / or diabetic patients with regular physical exercise. As a method, the cross-sectional epidemiological and crosssectional study of NCDs and drug use in individuals 60 years of age and older, regular exercise and diagnosed with Systemic Hypertension (HBV) and / or Diabetes Mellitus (DM) . A total of 223 elderly people were evaluated, being $14.8 \%(n=33)$ males and $85.2 \%(n=$ 190) females. The mean age was $69.8 \pm 6.9$ years. In relation to the weekly frequency of the exercises, it was observed that $57.8 \%$ practiced them 1 to 2 times a week; 33.6\%, from 3 to 4 times; and 8.5\%, from 5 to 7 times a week. Regarding drug use, 142 types were reported, among them antihypertensives, lipid-lowering drugs, antidepressants, cerebral psychotropics, hypoglycemic agents and suppressors of bone absorption. Among the DNCTs investigated, DM, SAH, dyslipidemias and hypothyroidism were the most prominent. The correlation between exercise time and drug numbers showed a strong statistical correlation $(r=0.0848)$, whereas for NCDs, this correlation was moderate $(r=0.0593)$. It was concluded that, with aging, there is an increase in the prevalence of $C N C D$, a decrease in the practice of physical exercise, and an increase in the consumption of drugs. It was demonstrated a greater female adherence in the practice of physical exercise, but the greater the age, the lower the adhesion. and the elderly practicing physical exercise for a longer time consumed fewer drugs and had less $C N C D$.

Keywords: Elderly; Exercise; Chronic disease; Use of medications.

RESUMEN: El objetivo fue analizar la incidencia de enfermedades crónicas no transmisibles (DCNT) y la utilización de fármacos en ancianos hipertensos y / o diabéticos practicantes regulares de ejercicio físico. Como método, el estudio de campo, epidemiológico y transversal sobre DCNT y la utilización de fármacos en indivíduos de 60 años o más de edad, practicantes regulares de ejercicio físico y diagnosticados con Hipertensión Arterial Sistémica (HAS) y / o Diabetes Mellitus (DM). Se evaluaron 223 ancianos, siendo el 14,8\% $(n=33)$ del sexo masculino y el 85,2\% $(n=190)$ del sexo femenino. La edad media fue de 69,8 \pm 6,9 años. En relación a la frecuencia semanal de los ejercicios, se observó que el 57,8\% los practicaban de 1 a 2 veces por semana; 33,6\%, de 3 a 4 veces; y el 8,5\%, de 5 a 7 veces a la semana. En cuanto al uso de fármacos, 142 tipos fueron relatados, entre ellos, se destacaron los antihipertensivos, hipolipemiantes, antidepresivos, psicotrópicos cerebrales, hipoglucemiantes y supresores de absorción ósea. Entre las DNCT investigadas, se destacaron el DM, la HAS, las dislipidemias y el hipotiroidismo. En la correlación entre el tiempo de ejercicio físico y el número de fármacos se pudo observar una fuerte correlación estadística $(r=0,0848)$, mientras que para las DCNT, esta correlación fue moderada $(r=$ 0,0593). Se concluyó que, con el envejecimiento, se observa un aumento de la prevalencia de DCNT, una disminución de la práctica de ejercicio físico, y un aumento en el consumo de fármacos. Se demostró una mayor adhesión del sexo femenino en la práctica de ejercicio físico, pero cuanto mayor la edad, menor la adhesión. y los ancianos practicantes de ejercicio físico por más tiempo consumían menos fármacos y presentaban menos DCNT.

Palabras clave: Ancianos; Ejercicio; Enfermedad crónica; Uso de medicamentos. 


\section{Introdução}

O perfil populacional mundial está mudando, e gerando novos desafios aos profissionais da área da saúde. Estima-se que, em 2030, haverá mais idosos do que crianças e adolescentes no mundo, com expectativas de um percentual de 28,8\% de idosos (IBGE, 2009; Veras, 2012). Diante deste novo cenário, há novos desafios a serem cumpridos, visto que a demanda em saúde aumentará consideravelmente (Veras, 2012; Ribeiro, Alves, \& Meira, 2009), já que, normalmente, essa população é acometida por doenças crônicas não transmissíveis (DCNT) (IBGE, 2009).

As DCNT requerem um constante acompanhamento em saúde, pois representam diversos fatores de risco, com graus de acometimento variados, podendo acarretar em incapacidades ou até mesmo morte do indivíduo (Coelho, \& Burini, 2009). Estudos demonstram que, no ano de 2003, 29,9\% da população apresentavam, pelo menos, uma DCNT, porém, entre idosos, estes índices representavam cerca de 75,5\% (IBGE, 2009).

Essas enfermidades afetam diretamente a qualidade de vida do idoso, que necessita se adaptar perante tais prejuízos, causando limitação nas suas atividades, além de mudanças na expectativa de vida e impacto econômico negativo para o indivíduo (Alves, et al., 2007; Taddeo, et al., 2012; Malta, et al., 2014).

Destacam-se, entre as DCNT, a hipertensão arterial sistêmica (HAS), o diabetes mellitus (DM), as dislipidemias e as doenças osteomioarticulares como a osteoporose, osteartrite, artrite, algias de coluna e outras (Araújo, \& Lira, 2013; Silva, Feldman, Lima, Nobre, \& Domingues, 2006); Campolina, Adami, Santos, \& Lebrão, 2013).

Com referência à HAS, o quadro clínico, juntamente com a terapia medicamentosa, modificam as atividades cotidianas de um indivíduo, interferindo emocional e socialmente, afetando, também, sua qualidade de vida. Acomete cerca de 50\% a 70\% dos idosos, tornandose um dos principais fatores de mortalidade, sendo um fator de risco para as doenças cardiovasculares (Andrade, et al., 2014).

As doenças cardiovasculares, amputação de membros inferiores, e cegueiras, são algumas das complicações que a DM pode causar, além de ter um alto índice de mortalidade. É estimado que, em 2025, 5,4\% da população mundial terá DM (Toscano, 2004). Esta alta incidência ocorre pelas mudanças no estilo de vida, em que os indivíduos estão mais sedentários e com um aumento do consumo de dieta rica em calorias e gorduras. O indivíduo com DM tem uma tendência maior em desenvolver HAS, ambas sendo grande problema de saúde pública em todo o país (Taddeo, et al., 2012; Toscano, 2004). 
Com o aumento da longevidade e, na mesma proporção, o aumento das DCNT afetando a população idosa, surge a preocupação com o impacto causado na qualidade de vida e na independência destes sujeitos, visto que há diminuição da mobilidade e aumento no consumo de fármacos (Junior, et al., 2006; Pacheco, SCS, Santos, \& Pacheco, CRS, 2013).

Estudos demonstram que a polifarmácia em idosos é maior, e há um aumento nos índices de automedicação. Esta alta ingestão de fármacos acarreta alguns prejuízos, como tratamentos inadequados, admissões hospitalares agudas, interações medicamentosas, acúmulo de substâncias toxicas no organismo, reações adversas, entre outros problemas (Galato, \& Tiburcio, 2010; Nascimento, Chaves, Grossi, \& Lottenberg, 2010; Santos, Paes, Junior, Pacheco, SCS, \& Pacheco, CRS (2012).

A prática da polifarmácia entre os idosos, muitas vezes, se torna necessária pelo acometimento de DCNT e múltiplos sintomas, visto que essa prática não indica que a prescrição de vários fármacos de uso contínuo esteja incorreta, já que são, por muitas vezes, necessários para garantir uma melhor qualidade de vida para o idoso (Santos, et al., 2013). No entanto, há pioras nas condições de saúde física e mental entre os idosos com a prática contínua da polifarmácia (Neves, et al., 2013).

Os exercícios físicos regulares contribuem para a modificação no estilo de vida, proporcionam menores incidências de DCNT, e promovem maior qualidade de vida aos idosos (Araújo, \& Lira, 2013; Ciolac, \& Guimarães, 2004). Por meio do condicionamento físico, pode-se melhorar o estado físico e mental (Araújo, \& Lira, 2013), a massa óssea e muscular, a força muscular global, coordenação, equilíbrio, sistema imunológico e autoestima, diminuindo a dependência funcional (Nogueira, Santos, Alverne, Martins, \& Magalhães, 2012; Faria, \& Santos, 2011).

\section{Objetivo}

Analisar a incidência de DCNT e utilização de fármacos de idosos hipertensos e/ou diabéticos praticantes regulares de exercício físico, e correlacionar o tempo de prática dos exercícios físicos regulares com a incidência das DCNT e com a utilização de fármacos. 


\section{Métodos}

Trata-se de um estudo de campo, epidemiológico e transversal sobre a utilização de fármacos e DCNT em idosos hipertensos, diabéticos e/ou hipertensos e diabéticos praticantes regulares de exercício físico.

A abordagem foi direta, realizada nas Unidades Básicas de Saúde (UBS) e nas Estratégias de Saúde Família (ESF), onde se realizavam os exercícios físicos referentes ao Programa Agita Assis-SP. Tal programa é coordenado pela Secretaria Municipal de Saúde do município de Assis, com o intuito de combater o sedentarismo e divulgar a importância do exercício físico $^{23}$. Cada grupo é supervisionado por um educador físico que desenvolve exercícios aeróbicos, funcionais e caminhadas com uma frequência de dois dias semanais em cada unidade de atendimento, com duração de, aproximadamente, sessenta minutos, no período da manhã.

Esta pesquisa foi aprovada pelo Comitê de Ética em Pesquisa envolvendo seres humanos (parecer n. ${ }^{\circ}$ 752.853), com consentimento da referida Secretaria Municipal da Saúde. A coleta dos dados ocorreu entre o mês setembro/2014 a março/2015.

A pesquisa foi anuída pela coordenadora do projeto e, após assinado o termo de compromisso, iniciou-se a coleta de dados nas dezenove unidades do programa.

Cada idoso autorizou sua participação mediante ao Termo de Consentimento Livre e Esclarecido (TCLE). A entrevista aconteceu de forma individual, onde havia questões a respeito de uso de fármacos, doenças crônicas, dados socioeconômicos, identificação e informações pessoais. O questionário foi baseado no estudo denominado Censo das Pessoas com Deficiência no município de Assis, SP (Santos, Pacheco, SCS, \& Pacheco, CRS).

Foram admitidos para o estudo idosos de ambos os sexos, cadastrados no programa Agita Assis por, no mínimo, seis meses, e independentes funcionalmente.

Adotou-se como critério de exclusão: idosos que apresentassem doenças restritivas que interferiam no movimento e que utilizem dispositivos auxiliares para a marcha.

Os dados coletados foram digitados e sistematizados em banco de dados do Excel $^{\circledR}$, sendo analisados por meio de estatística descritiva simples, utilizando-se de média, desvio padrão e porcentagem, sendo apresentados por amostras estratificadas por idade: 60 a 69 anos, grupo 1 (G1); 70 a 79 anos, grupo 2 (G2); e 80 anos ou mais, grupo 3 (G3). Para comparação estatística entre os grupos, utilizou-se o software GraphPad Prism $5^{\circledR}$, por meio do teste não paramétrico de Krushall Wallis, considerando significância de 5\% (p<0,05). 


\section{Resultados}

A amostra deste estudo foi de 223 idosos, sendo 14,8\% ( $n=33)$ do sexo masculino e 85,2\% (n=190) do sexo feminino, com média de 69,8 8 6,9 anos de idade (mínima de 60 anos e máxima de 88 anos). Na Figura 1, visualizam-se as categorias estratificadas (G1, G2 e G3) por idade e sexo da amostra.

Figura 1. Sexo e idade de idosos hipertensos e/ou diabéticos praticantes regulares de exercício físico

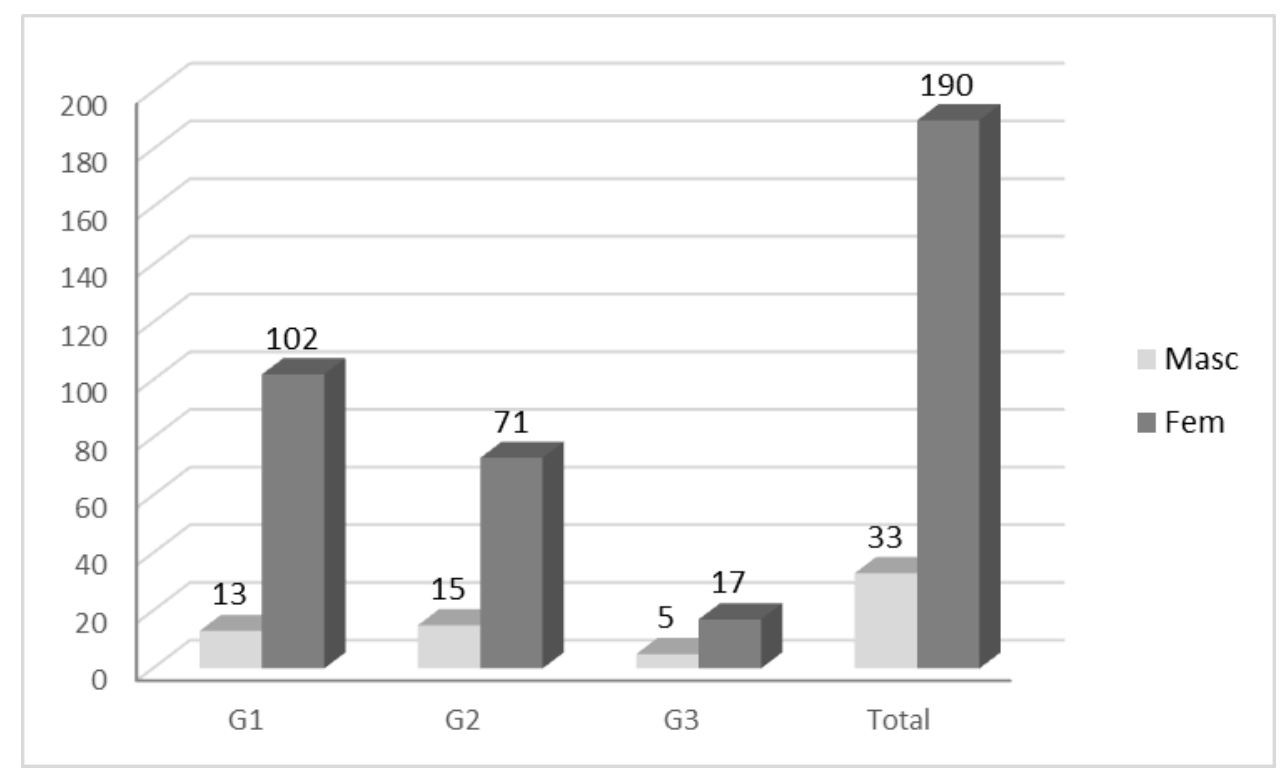

Quanto à variação dos sujeitos por grupos, observou-se que, quanto maior a idade, menor a amostra em ambos os sexos, sendo que, na comparação das categorias masculinas e femininas, esses valores são significativos $(\mathrm{p}<0,0001)$.

O tempo de adesão aos exercícios físicos regulares demonstrou uma curva ascendente até os dez anos de prática, e uma queda após esse período, conforme visualizado na Figura 2. 
Figura 2. Tempo de exercício físico praticado regularmente pelos idosos hipertensos e/ou diabéticos

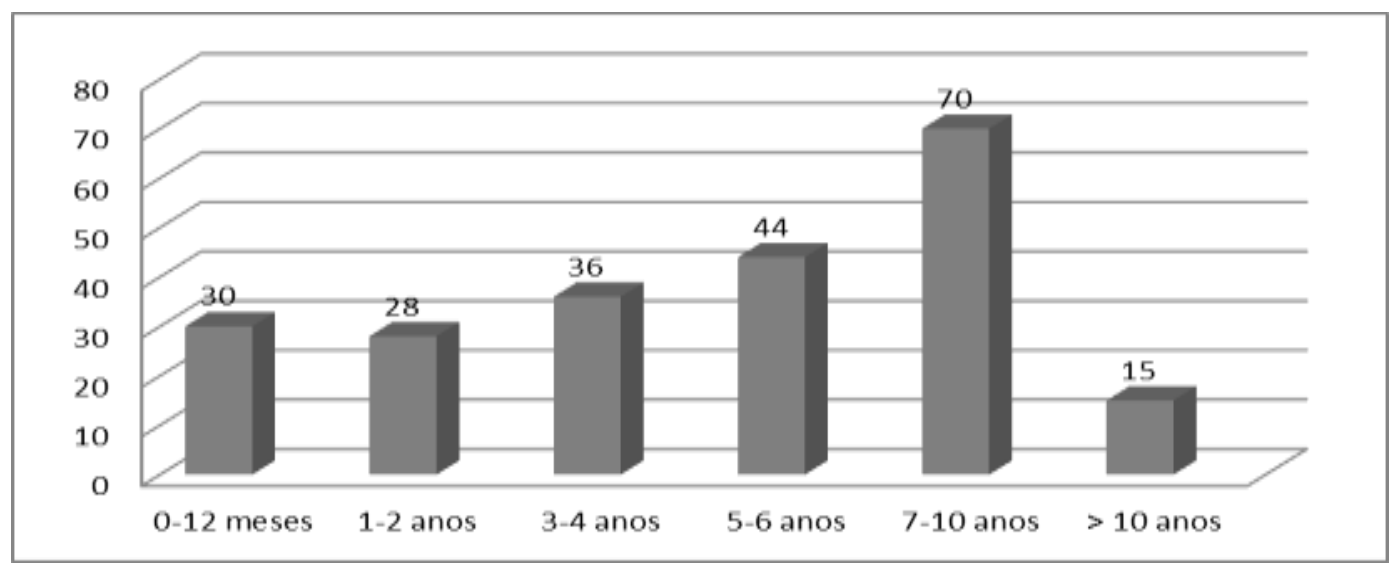

Já em relação à frequência semanal dos exercícios, observou-se que 57,8\% dos idosos praticavam exercícios regulares de 1 a 2 vezes por semana, 33,6\% de 3 a 4 vezes na semana e $8,5 \%$ de 5 a 7 vezes na semana.

Quanto ao uso de fármacos, observaram-se duas situações: a primeira relacionada aos idosos praticantes regulares de exercício físico que relataram não possuir DCNT (n=26), e destes, 23,8\% ( $\mathrm{n}=6$ ) faziam uso de medicamentos; a segunda é a dos idosos que relataram possuir alguma(s) DCNT (n=197), e destes, 87,30\% (n=172) faziam uso de fármacos.

Dos idosos que possuíam DCNT, e relataram não fazer uso de medicamentos, observou-se que as doenças auto relatadas mais frequentes foram: DM, HAS, dislipidemias e hipotireoidismo, associadas ou não.

Quanto aos tipos de fármacos utilizados, verificou-se que houve uma variedade de categorias, conforme as doenças (quadro 1).

Foram catalogados 142 tipos de medicamentos, destacando-se para os seguintes grupos: anti-hipertensivos, hipolipidemiantes, antidepressivos, psicotrópicos cerebrais, hipoglicemiantes e supressores de absorção óssea. 
Quadro 1. Categorias de fármacos por disfunção clínica dos idosos hipertensos e/ou diabéticos praticantes regulares de exercício físico

\begin{tabular}{|c|c|c|}
\hline $\begin{array}{l}\text { Disfunção } \\
\text { clínica }\end{array}$ & $\begin{array}{l}\text { Categorias } \\
\text { de fármacos }\end{array}$ & $\begin{array}{c}\text { Número } \\
\text { de medicamentos }\end{array}$ \\
\hline \multirow[t]{5}{*}{ Cardiovasculares } & Anti-hipertensivos & 39 \\
\hline & Cardiovasculares & 7 \\
\hline & Antianginosos & 3 \\
\hline & Antiarrítmicos & 3 \\
\hline & Antivaricosos & 1 \\
\hline \multirow[t]{4}{*}{ Metabólicos } & Hipolipidemiantes & 11 \\
\hline & Hiperglicêmicos & 9 \\
\hline & Tireoidianos & 7 \\
\hline & Repositores hormonais & 3 \\
\hline \multirow[t]{6}{*}{ Sistema nervoso central } & Ansiolíticos / Tranquilizantes & 2 \\
\hline & Sedativos / Calmantes & 2 \\
\hline & Antidepressivos & 11 \\
\hline & Psicotrópicos cerebrais & 10 \\
\hline & Antivertiginosos & 3 \\
\hline & Fitoterápicos & 1 \\
\hline \multirow[t]{4}{*}{ Sistema musculoesquelético } & Anti-inflamatórios/Antirreumáticos & 7 \\
\hline & Analgésicos & 1 \\
\hline & Supressores de absorção óssea & 9 \\
\hline & Complexos vitamínicos & 3 \\
\hline \multirow[t]{4}{*}{ Outros } & Sistema gastrointestinal & 6 \\
\hline & Antibióticos & 1 \\
\hline & Antineoplásicos & 2 \\
\hline & Sistema oftalmológico & 1 \\
\hline TOTAL & & 142 \\
\hline
\end{tabular}

Na correlação entre o tempo de exercício físico e o número de fármacos, pôde-se observar forte correlação estatística $(r=0,0848)$, enquanto que, para as DCNT, foi moderada $(\mathrm{r}=0,0593)$.

\section{Discussão}

Observou-se, neste estudo, uma considerável adesão do sexo feminino (85,2\%) ao exercício físico. Esta evidência é amplamente verificada na literatura científica (Ribeiro, et al., 2012; Cavalli, et al., 2014; Medeiros, Streit, Sandreschi, Fortunato, \& Mazo, 2014). 
Ribeiro, et al. (2012) realizaram uma pesquisa com participantes do Núcleo de Atividade para a Terceira idade (NATI), na cidade de Pelotas, RS, com idosos que realizaram pelo menos uma atividade oferecida pelo programa. Obteve-se uma amostra total de 199 idosos de ambos os sexos, sendo 4 (2\%) do sexo masculino e 195 (98\%) do sexo feminino.

No estudo de Cavalli, et al. (2014), a amostra foi composta por 263 participantes de ambos os sexos, em que se avaliaram diferenças e semelhanças entre idosos de programas de exercício físico gratuito de duas universidades conveniadas, sendo uma no Brasil e a outra em Portugal. Do mesmo modo, houve 89,4\% de aderência aos programas pelo sexo feminino, evidenciando, assim, a maior adesão das mulheres praticantes dos grupos de exercício físico comparadas aos homens.

Sabe-se que os homens apresentam maior resistência aos métodos preventivos e de atenção primária à saúde. Já as mulheres têm uma preocupação maior com o aparecimento de doenças e estão mais atentas aos sinais que o corpo apresenta, passam com maior frequência em consultas médicas, mantendo, assim, os cuidados primários de atenção à saúde (Ribeiro, et al., 2012; Cavalli, et al., 2014; Medeiros, Streit, Sandreschi, Fortunato, \& Mazo, 2014). Nesta perspectiva, torna-se importante a implantação de programas que enfatizem os benefícios do exercício físico, principalmente para o sexo masculino.

A prevenção dos problemas de saúde, a recomendação médica, a socialização e o prazer são os principais fatores que motivam os idosos a participar de exercícios físicos (Meurer, Benedetti, \& Mazo, 2012). Por outro lado, a falta de companhia, falta de tempo e desconhecimento sobre os benefícios são fatores que dificultam a prática de exercícios (Cavalli, et al., 2014).

A prática de exercícios físicos na terceira idade previne e combate os prejuízos que podem ser causados pelo sedentarismo que, juntamente com o processo do envelhecimento, propiciam o surgimento de doenças crônicas (Vila, Silva, Simas, Guimarães, \& Parcias, 2013).

O estudo de Silva, Goulart, Lanferdini, e Marcon (2012) comparou pessoas idosas com sedentárias, e demonstrou que idosos praticantes de exercício físico apresentam melhor qualidade de vida, capacidade funcional, estado geral de saúde, boa capacidade de locomoção e satisfação com seu sono.

O presente estudo concorda com Santos, Paes, Junior, Pacheco, SCS, e Pacheco, CRS (2012) que, quanto maior a idade, menor é a adesão a programas de exercícios físicos supervisionados. 
O estudo de Ribeiro, et al. (2012) também corrobora esse achado. Eles observaram que $29,6 \%$ dos indivíduos que realizavam exercícios físicos estavam no estrato de 65 a 69 anos, sendo que, dentre aqueles que apresentavam idade superior a 80 anos, apenas $8,1 \%$ aderiam a essa prática.

Costa, \& Neri (2011) constataram que a prevalência do sedentarismo é maior em idosos com 80 anos ou mais de idade. Concluíram que as doenças crônicas têm uma contribuição importante para esse achado.

Uma das questões observadas é o tempo de adesão ao programa, já que a maioria $(\mathrm{n}=70)$ participa dos exercícios de 7 a 10 anos. Isso demonstra uma satisfação com o programa de exercícios e conscientização da importância regular da prática de exercício físico.

Quanto à prática semanal, 57,8\% dos idosos, praticavam exercício físico de 1 a 2 vezes na semana. De acordo com Moraes, et al. (2011), o exercício físico com frequência semanal reduzida pode trazer vários benefícios como a redução da glicemia e da pressão arterial. Neto, e Castro (2012) relataram que o exercício físico regular tem demonstrado prevenir o aumento da pressão arterial associado à idade e diminuir a chance de pessoas com risco aumentado de desenvolvê-la.

No presente estudo, foi observado que $12,70 \%$ dos sujeitos que possuem alguma DCNT, não fazem uso de nenhuma medicação. Carvalho, Leopoldino, e Silva (2012) relataram que um dos motivos pelo qual ocorre a não adesão ao tratamento medicamentoso é o fator assintomático das DCNT. Uma pesquisa com usuários da Atenção Primária do Sistema Único de Saúde mostrou que $35,4 \%$ da população estudada não aderiu ao tratamento farmacológico (Junior, Lindner, \& Helena, 2013).

O estudo realizado por Galato, e Tiburcio (2010), sobre medicamentos, apontou que o fármaco mais utilizado é o anti-hipertensivo, equivalendo a 63,5\%. Gonçalves, Goersch, Lamboglia, \& Lima (2013), em seu estudo sobre DCNT e fármacos, também confirmaram, como neste estudo, que o fármaco mais utilizado por idosos são os anti-hipertensivos.

É comum a utilização de cinco ou mais medicamentos entre os idosos, sendo que a polifarmácia entre mulheres é maior (Santos, et al., 2013). A relação entre polifarmácia e faixas etárias mais avançadas é outro fator importante, sendo uma consequência das diversas doenças crônicas e dos múltiplos sintomas dos idosos (Santos, et al., 2013). 
Uma importante consideração metodológica foi a grande dificuldade de os idosos relatarem, espontaneamente, as possíveis doenças e os medicamentos utilizados, sendo que, para facilitar a coleta, utilizou-se um roteiro de perguntas pré-estabelecidas.

\section{Conclusão}

Com o envelhecimento, observa-se um aumento da prevalência de DCNT, uma diminuição da prática de exercício físico e, consequentemente, um aumento no consumo de fármacos.

Contudo, foi demonstrada uma maior adesão do sexo feminino na prática de exercício físico; que, quanto maior a idade, menor a adesão, e os idosos que praticavam exercício físico por mais tempo consumiam menos fármacos e, relativamente, apresentavam menos DCNT.

\section{Referências}

Alves, L. C., Leimann, B. C. Q., Vasconcelos, M. E. L., Carvalho, M. S., Vasconcelos, A. G. G., Fonseca, T. C. O., Lebrão, M. L., \& Laurenti, R. (2007). A influência das doenças crônicas na capacidade funcional dos idosos no município de São Paulo, Brasil. Rio de Janeiro, RJ: Cad. Saúde Pública, 23(8), 1924-1930. Recuperado em 20 maio, 2015, de: http://dx.doi.org/10.1590/S0102-311X2007000800019.

Andrade, J. M. O., Rios, L. R., Teixeira, L. S., Vieira, F. S., Mendes, D. C., Vieira, M. A., \& Silveira, M. F. (2014). Influência de fatores socioeconômicos na qualidade de vida de idosos hipertensos. Ciência e Saúde Coletiva, 19(8), 3497-3504. Recuperado em 20 maio, 2015, de: doi: 10.1590/1413-81232014198.19952013.

Araújo, M. V. G. G., \& Lira, F. A. S. (2013). Atividade física na redução da hipertensão arterial em idosos na unidade da saúde da família. REBES Revista Brasileira de Educação e Saúde, 3(4), 1-10. Recuperado em 20 maio, 2015, de: http://www.gvaa.com.br/revista/index.php/REBES/article/viewFile/2391/1940.

Assis News. (2013). Agita Assis promove show de talentos no teatro municipal [internet]. http://www.assisnews.com.br/editoriais/geral/2013/10/agita-assis-promove-show-de-talentosno-teatro-municipal.html.

Campolina, A. G., Adami, F., Santos, J. L. F., \& Lebrão, M. L. (2013). A transição de saúde e as mudanças na expectativa de vida saudável da população idosa: possíveis impactos da prevenção de doenças crônicas. Cad. Saúde Pública, 29(6), 1217-1729. Recuperado em 20 maio, 2015, de: http://dx.doi.org/10.1590/S0102-311X2013000600018. 
Carvalho, A. L. M., Leopoldino, R. W. D., \& Silva, J. E. G. (2012). Adesão ao tratamento medicamentoso em usuários cadastrados no programa Hiperdia no município de Terezina (PI). Ciência e Saúde Coletiva, 17(7), 1885-1892. Recuperado em 20 maio, 2015, de: http://dx.doi.org/10.1590/S1413-81232012000700028.

Cavalli, A. S., Pogorzelski, L. V., Domingues, M. R., Afonso, M. R., Ribeiro, J. A. B., \& Cavalli, M. O. (2014). Motivação de pessoas idosas para a prática de atividade física: estudo e comparativo entre dois programas universitários-Brasil e Portugal. Rio de Janeiro, RJ: Rev. Bras. Geriatr. Gerontol, 17(2), 255-264. Recuperado em 20 maio, 2015, de: http://dx.doi.org/10.1590/S1809-98232014000200004.

Ciolac, E. G., \& Guimarães, G. V. (2004). Exercício Físico e Síndrome Metabólica. Rev. Bras. Med. Esporte, 10(4), 318-325. Recuperado em 20 maio, 2015, de: http://www.scielo.br/pdf/\%0D/rbme/v10n4/22048.

Coelho, C. F., \& Burini, R. C. (2009). Atividade física para prevenção e tratamento das doenças crônicas não transmissíveis e da incapacidade funcional. Campinas, SP: Rev. Nutr., 22(6), 937-946. Recuperado em 20 maio, 2015, de: http://dx.doi.org/10.1590/S141552732009000600015.

Costa, T. B., \& Neri, A. L. (2011). Medidas de atividade física e fragilidade em idosos: dados do FIBRA Campinas, São Paulo, Brasil. Rio de Janeiro, RJ: Cad. Saúde Pública, 27(8), 15371550. Recuperado em 20 maio, 2015, de: http://dx.doi.org/10.1590/S0102311 X2011000800009.

Faria, L., \& Santos, L. A. C. (2011). As profissões de saúde: uma análise crítica do cuidar. Rio de Janeiro, RJ: História, Ciência, Saúde - Manguinhos, 18(1), 227-240. Recuperado em 20 maio, 2015, de: http://dx.doi.org/10.1590/S0104-59702011000500012.

Galato, D., Silva, E. S., \& Tiburcio, L. S. (2010). Estudo de utilização de medicamentos em idosos residentes em uma cidade do Sul de Santa Catarina (Brasil): um olhar sobre a polimedicação. Ciência e Saúde Coletiva, 15(6), 2899-2905. Recuperado em 20 maio, 2015, de: http://dx.doi.org/10.1590/S1413-81232010000600027.

Gonçalves, F. T. R., Goersch, C. M., Lamboglia, F., \& Lima, D. L. F. (2013). Prevalência de doenças crônicas e uso de fármacos em idosos praticantes de exercício físico supervisionado. Fortaleza, CE: Rev. Bras. Promoc. Saúde, 26(3), 372-380. Recuperado em 20 maio, 2015, de: http://www.redalyc.org/pdf/408/40829885010.pdf.

IBGE. (2009). Instituto Brasileiro de Geografia e Estatística. Indicadores Sociodemográficos e de Saúde no Brasil. Estudo e pesquisa informação demográfica e socioeconômica número 25. Rio de Janeiro, RJ.

Júnior, A. A. S., Lindner, S., \& Helena, E. T. S. (2013). Avaliação da adesão terapêutica em idosos atendidos na prevenção primária. Rev. Assoc. Med. Bras., 59(6), 614-621. Recuperado em 20 maio, 2015, de: http://www.ufjf.br/pgsaudecoletiva/files/2016/12/PREVAL\%C3\%8ANCIA-DE-USO-DEBENZODIAZEP\%C3\%8DNICOS-EM-IDOSOS-E-FATORES-.pdf.

Junior, D. P. L., Amaral, R. T., Veiga, E. V., Cárnio, E. C., Nogueira, M. S., \& Pelá, I. R. A (2006). Farmacoterapia no idoso: Revisão sobre a abordagem multiprofissional no controle da hipertensão arterial sistêmica. Rev. Latino-Am Enfermagem, 14(3), 428-434. Recuperado em 20 maio, 2015, de: https://www.revistas.usp.br/rlae/article/viewFile/2315/2455. 
Malta, D. C., Gosch, C. S., Buss, P., Rocha, D. G., Rezende, R., Freitas, P. C., et al. (2014). Doenças crônicas não transmissíveis e o suporte das ações intersetoriais no seu enfrentamento. Ciência e Saúde Coletiva, 19(11), 4341-4350. Recuperado em 20 maio, 2015, de: doi: 10.1590/1413-812320141911.07712014.

Medeiros, P. A., Streit, I. A., Sandreschi, P. F., Fortunato, A. R., \& Mazo, G. Z. (2014). Participação masculina em modalidades de atividade física de um programa para idosos: um estudo longitudinal. Ciência e Saúde Coletiva, 19(8), 3479-3488. Recuperado em 20 maio, 2015, de: doi: 10.1590/1413-81232014198.16252013.

Meurer, S. T., Benedetti, T. R. B., \& Mazo, G. Z. (2012). Fatores motivacionais de idosos praticantes de exercício físico: um estudo baseado na teoria da autoderminação. Estudos de Psicologia, 17(2), 299-303. Recuperado em 20 maio, 2015, de: http://www.scielo.br/pdf/epsic/v17n2/14.pdf.

Moraes, W. M., Souza, P. R. M., Pinheiro, M. H. N., Trigoyen, M. C., Medeiros, A., \& Koike, M. K. (2011). Programa de exercícios físicos baseado em frequência semanal mínima: efeitos na pressão arterial e aptidão física em idosos hipertensos. São Carlos, SP: Rev. Bras. Fisioter., Recuperado em 20 maio, 2015, de: http://dx.doi.org/10.1590/S141335552012005000013.

Nascimento, A. B., Chaves, E. C., Grossi, S. A. A., \& Lottenberg S. A. (2010). A relação entre polifarmácia, complicações crônicas e depressão em portadores de Diabetes Mellitus tipo 2. Rev. Esc. Enfermagem USP, 44(1), 40-46. Recuperado em 20 maio, 2015, de: http://www.scielo.br/pdf/reeusp/v44n1/a06v44n1.pdf.

Neto, M. G., \& Castro, M. F. (2012). Estudo Comparativo da independência funcional e qualidade de vida entre idosos ativos e sedentários. Rev. Bras. Med. Esporte, 18(4). Recuperado em 20 maio, 2015, de: http://dx.doi.org/10.1590/S1517-86922012000400003.

Neves, S. J. F., Marques, A. P. O., Leal, M. C. C., Diniz, A. S., Medeiros, T. S., \& Arruda, I. K. G. (2013). Epidemiologia do uso de medicamentos entre idosos em área urbana do Nordeste do Brasil. Rev. Saúde Pública, 47(4), 759-768. Recuperado em 20 maio, 2015, de: http://dx.doi.org/10.1590/S0034-8910.2013047003768.

Nogueira, I. C., Santos, Z. M. S. A., Alverne, D. G. B. M., Martins, A. B. T., \& Magalhães, C. B. A. (2012). Exercício Físico no controle da hipertensão arterial em idosos: uma revisão sistemática. Rev. Bras. Geriatr. Gerontol., 15(3), 587-601. Recuperado em 20 maio, 2015, de: http://dx.doi.org/10.1590/S1809-98232012000300019.

Pacheco, S. C. S., Santos, B. M., \& Pacheco, C. R. S. (2013). Independência funcional: perfil das pessoas acometidas por Acidente Vascular Encefálico. Arq Ciênc Saúde, 20(1), 17-21. Recuperado em 20 maio, 2015, de: http://repositorio-racs.famerp.br/racs_ol/vol-201/ID_522_(Volume_20(1)_2013_jan-mar.pdf.

Ribeiro, J. A. B., Cavalli, A. S., Cavalli, M. O., Pogorzelski, L. V., Prestes, M. R., \& Ricardo, L. I. C. (2012). Adesão de idosos a programas de atividade física: Motivação significância. Florianopolis, SC: Rev. Bras. Ciênc. Esporte, 34(4), 969-984. Recuperado em 20 maio, 2015, de: http://www.scielo.br/pdf/rbce/v34n4/v34n4a12.

Ribeiro, L. C. C., Alves, P. B., \& Meira, E. P. (2009). Percepção dos Idosos sobre as alterações fisiológicas do envelhecimento. Ciênc. Cuid. Saúde, 8(2), 220-227. Recuperado em 20 maio, 2015, de: doi: 10.4025/cienccuidsaude.v8i2.8202. 
Santos, B. M., Pacheco, S. C. S., \& Pacheco, C. R. S. (2010). Retrato das pessoas com deficiência no município de Assis, SP. In: $3^{\circ}$ Congresso Nacional de Diversidade e Inclusão CONADI. São José dos Campos, SP. $3^{\circ}$ CONADI: Tecnologia e Trabalho.

Santos, B. M., Paes, H. H. F., Junior, P. R. R., Pacheco, S. C. S., \& Pacheco, C. R. S. (2012). Doenças fármacos e terapêutica em idosos com deficiência. J Health Sci Inst., 30(2), 181-185. Recuperado em 20 maio, 2015, de: https://www.unip.br/comunicacao/publicacoes/ics/edicoes/2012/02_abr-jun/ V30 _n2_2012_p181-185.pdf.

Santos, T. R. A., Lima, D. M., Nakatani, A. Y. K., Pereira, L. V., Leal, G. S., \& Amaral, R. G. (2013). Consumo de Medicamentos por idosos, Goiânia, Brasil. Rev. Saúde Pública, 47(1), 94-103. Recuperado em 20 maio, 2015, de: http://www.scielo.br/pdf/rsp/v47n1/13.pdf.

Silva, M. F., Goulart, N. B. A., Lanferdini, F. J., Marcon, M., \& Dias, C. P. (2012). Relação entre os níveis de atividade física e qualidade de vida de idosos sedentários e fisicamente ativos. Rio de Janeiro, RJ: Rev Bras. Geriatr. Gerontol, 15(4), 635-642. Recuperado em 20 maio, 2015, de: http://dx.doi.org/10.1590/S1809-98232012000400004.

Silva, T. R., Feldman, C., Lima, M. H. A., Nobre, M. R. C., \& Domingues R. Z. L. (2006). Controle de diabetes mellitus e hipertensão arterial com grupos de intervenção educacional e terapêutica em seguimento ambulatorial de uma unidade básica de saúde. Saúde e sociedade, 15(3), 180-189. Recuperado em 20 maio, 2015, de: http://dx.doi.org/10.1590/S010412902006000300015.

Taddeo, P. S., Gomes, K. W. L., Caprara, A., Gomes, A. M. A., Oliveira, G. C., \& Moreira, T. M. M. (2012). Acesso, prática educativa e empoderamento de pacientes com doenças crônicas. Ciência e Saúde Coletiva, 17(11), 2923-2930. Recuperado em 20 maio, 2015, de: https://www.scielosp.org/article/csc/2012.v17n11/2923-2930/pt/.

Toscano, C. M. (2004). As Campanhas nacionais para detecção das doenças crônicas não transmissíveis: diabetes e hipertensão arterial. Ciência Saúde Coletiva, 9(4), 885-895. Recuperado em 20 maio, 2015, de: http://dx.doi.org/10.1590/S1413-81232004000400010.

Veras, R. P. (2012). Gerenciamento de doença crônica: Equívoco para o grupo etário dos idosos. Rev. Saúde Pública, 46(6), 929-934. Recuperado em 20 maio, 2015, de: http://dx.doi.org/10.1590/S0034-89102012000600001.

Vila, C. P., Silva, M. E. M., Simas, J. P. N., Guimarães, A. C. A., \& Parcias, S. R. (2013). Aptidão física funcional e nível de atenção em idosas praticantes de exercício físico. Rio de Janeiro, RJ: Rev. Bras. Geriatr. Gerontol., 16(2), 355-364. Recuperado em 20 maio, 2015, de: http://dx.doi.org/10.1590/S1809-98232013000200015.

Recebido em 30/12/2015

Aceito em 30/01/2016 
Giselly Cândido - Fisioterapeuta.

E-mail: prochajr@terra.com.br

Bruna Mastroldi dos Santos - Graduação em Fisioterapia, Universidade Paulista, Assis, SP. Especialização em Fisioterapia Geriátrica, Universidade Federal de São Carlos, UDSCar. Formação em Pilates, pelo Método STTOT PILATES. Mestrado em Ensino e Saúde, Faculdade de Medicina, FAMEMA, de Marília, SP. Atua como docente na Universidade Paulista, UNIP, Campus Assis.

E-mail: prochajr@terra.com.br

Paulo Roberto Rocha Júnior - Graduação em Fisioterapia, Faculdades Salesianas de Lins. Lins, SP. Especialização em Terapia Manual e Postural, Centro Universitário de Maringá, CESUMAR, Maringá, PR. Mestrado em Saúde Coletiva, UNESP, FMB. Doutorado em Saúde Coletiva, UNESP, FMB. Atualmente é professor das Faculdades Adamantinenses Integradas (FAI, Adamantina, SP, Departamentos de Fisioterapia e Gerontologia, da Universidade Paulista (UNIP, Assis, SP, Departamento de Fisioterapia); e do curso de Pós-Graduação em Saúde Coletiva, do Instituto Rhema de Ensino, Arapongas, PR. Tem experiência na área de Fisioterapia, atuando principalmente nos seguintes temas: Geriatria e Gerontologia, Fisioterapia Preventiva em Estratégia de Saúde da Família e Cuidadores de Idosos.

E-mail: prochajr@terra.com.br 